\title{
TELERRADIOLOGIA NO BRASIL: UMA BREVE REVISÃO HISTÓRICA
}

\author{
Teleradiology in Brazil: a brief historical review
}

\section{Claudio Marcio Amaral de Oliveira Lima ; Alair Augusto Sarmet dos Santos ${ }^{2}$; Alexandra Maria Vieira Monteiro ${ }^{3}$}

Resumo Telerradiologia emergiu em 1972 e, com a evolução tecnológica, tem mudado drasticamente, principalmente, em virtude das novas tecnologias para digitalização e compactação das imagens e o aumento na velocidade e na infraestrutura da Internet. A telerradiologia é, atualmente, uma realidade na medicina brasileira, com competitividade, riscos e benefícios em um mundo globalizado. Este artigo tem como objetivo apresentar uma revisão da história da introdução da telerradiologia no Brasil e descrever experiências exitosas para a tele-educação.

Palavras-chave: Telemedicina, Telerradiologia, Tele-educação.

Abstract Teleradiology emerged in 1972 and, with technological developments, have changed dramatically, mainly because of new technologies for digitization and compression of images and the increase in speed and Internet infrastructure. Teleradiology is currently a reality in Brazilian medicine with competitiveness, risks and benefits in a globalized world. This article aims to present a review of the history of the introduction of teleradiology in Brazil and describe successful experiences for tele-education.

Keywords: Telemedicine, Teleradiology, Tele-education.

\section{Introdução}

A emergência da telerradiologia ocorreu nos Estados Unidos em 19721, caracterizada por um esforço deliberado em termos de pesquisas e pelo ingresso de fundos federais de curto prazo. Entre 1972 e 1975, a National Aeronautics and Space Administration (NASA) implementou um projeto de telerradiologia na reserva indígena Papago, no Arizona, onde uma van equipada com um aparelho de raios- $X$ foi conectada a equipe médica do "Public Health Service Hospital". Alguns outros projetos foram timidamente desenvolvidos, mas interrompidos por suspensão dos financiamentos ${ }^{1,2}$. Nos anos 1970 e ganhando força na década de 1980, a atenção voltou-se para os computadores com amplo desenvolvimento, tanto em software quanto em hardware, o que vai impulsionar e possibilitar a disponibilização comercial da telerradiologia ${ }^{1-4}$.

Na década passada, a telerradiologia mudou drasticamente, principalmente em virtude da introdução de ferramentas de comunicação de baixo custo como a Internet, melhorias no custo-benefício (preço versus

1. Médico Radiologista da rede Labs D’Or. Rio de Janeiro, RJ, Brasil; 2. Professor Associado de Radiologia da Faculdade de Medicina da UFF e Chefe do Serviço de Radiologia do HUAP/UFF e do Hospital de Clinicas Niterói (HCN). Rio de Janeiro, RJ, Brasil; 3. Professora Associada de Radiologia da Faculdade de Ciências Médicas e Coordenadora do Laboratório de Telessaúde da UERJ. Rio de Janeiro, RJ, Brasil. 
desempenho) dos computadores, na ampla adoção do sistema de comunicação e arquivamento de imagens (Picture Archiving and Communications System - PACS) e de sistemas de informação e gerenciamento em radiologia (Radiology Information System - RIS). A utilização desses sistemas totalmente digitais oferece inúmeras vantagens para médicos radiologistas, médicos solicitantes, gestores, meio ambiente e qualificação no atendimento à pacientes ${ }^{2-4}$. Ademais, todo este arquivamento em dados e imagens radiológicas pode ser integrado ao prontuário eletrônico do paciente em sistemas como o Hospital Information System (HIS) ${ }^{2-5}$.

No Brasil as ações em telerradiologia vêm sendo realizadas, progressivamente, desde a década de 90, mas se intensificando nos últimos anos. Um país com dimensões continentais demanda soluções em telemedicina, sobretudo para áreas remotas. Muitos estudos $2,5-15$ foram feitos para avaliar os prós e contras dessa nova forma de abordagem em radiologia e diagnóstico por imagem e já há evidências científicas que demonstram a efetividade na relação custo-benefício 2,4,13-19.

\section{Telerradiologia no Brasil}

O Colégio Brasileiro de Radiologia (CBR) foi fundado em 15 de setembro de 1948, em São Paulo, durante a realização da primeira Jornada Brasileira de Radiologia com a missão de atuar como órgão representativo dos médicos especializados nas áreas de Radiologia e Diagnóstico por Imagem, Medicina Nuclear e Radioterapia.

Nesta perspectiva e no contexto mundial da globalização da telemedicina, o CBR criou, em 2005, a Comissão de Telerradiologia cujos objetivos foram estimular a teleintegração na perspectiva da boa prática e da ética em medicina, acompanhamento de ações públicas e privadas em telerradiologia, promover a integração com outros órgãos representativos que normatizam o uso das tecnologias para fins de saúde e fomentar a atualização e capacitação profissional utilizando novas metodologias para educação a distância.

Esta comissão está atuando em diferentes cenários nacionais e internacionais e participou, ativamente, na normatização, pelo Conselho Federal de Medicina (CFM), da Resolução de n 1890/2009 que definiu a telerradiologia como o exercício da Medicina ${ }^{20-22}$. Esta resolução, de forma sucinta, assegura a relação médicopaciente e inclui a obrigatoriedade do consentimento livre e esclarecido, assinado pelo paciente ou responsável, para a prática da telerradiologia.

No setor jurídico fica posto que a responsabilidade pelo ato médico é do médico radiologista presencial sendo o médico radiologista "a distância" corresponsável legalmente. Além disso, fatores como qualidade de imagem e/ou informações clínicas insuficientes para o médico teleconsultor não poderão servir como justificativa para relatórios técnicos inadequados, uma vez que é de livre decisão do médico a atuação como médico teleconsultor. Em relação à padronização do relatório técnico, é obrigatório que estejam claramente presentes a identificação do médico presencial e a do médico teleconsultor, que deverá ter, também, registro no Conselho Regional de Medicina onde ocorre a prestação de serviços a distância.

Em 13 de abril de 2010, entrou em vigor o Novo Código de Ética Médica Brasileiro ${ }^{23}$ que, em parágrafo único, define "O atendimento médico a distância nos moldes da Telemedicina ou de outro método, dar-se-á sob regulamentação do CFM (Resolução de n 1643/2003)24.

Esta readequação da regulamentação da prestação de serviço em telerradiologia está, também, acontecendo no cenário internacional. O American College of Radiology ${ }^{25}$, por exemplo, publicou em 2004, a ultima revisão das normas técnicas para Telerradiologia, inicialmente descritas em 1994, que, entre outras coisas, 
determina que o radiologista presencial e o consultor a distância deverão estar regularmente licenciados e credenciados pela equipe do hospital origem dos exames. Atualmente, nos Estados Unidos os radiologistas possuem uma licença para cada estado no qual eles recebam imagens e forneçam interpretações, bem como uma licença para o estado no qual ocorrem fisicamente as interpretações. Da mesma forma, são credenciados em cada hospital para as quais eles fornecem serviço ${ }^{26}$. O Colégio Interamericano de Radiologia ${ }^{27}$ redigiu, recentemente, um documento com recomendações sobre a boa prática da telerradiologia, visto tratar-se de relação inter-países. Esta mesma iniciativa está acontecendo no continente europeu coordenada pelo European College of Radiology.

\section{Tele-educação em radiologia e diagnóstico por imagem}

Novas práticas em telemedicina e telerradiologia estão, progressivamente, modificando paradigmas na educação continuada, na atualização e na recertificação profissional $^{6-9}$. Várias iniciativas estão sendo feitas pelo Colégio Brasileiro de Radiologia e pela Associação Médica Brasileira, inclusive, especificamente para a qualificação em radiologia médica ${ }^{5,6,20}$ através, principalmente, de cursos a distância.

Por outro lado, o governo federal implementou, em 2006, dois programas em telemedicina ${ }^{28,29}$ e telessaúde com abrangência nacional tendo como principal objetivo a qualificação profissional: A Rede Universitária de Telemedicina (RUTE) e o Programa Nacional Telessaúde Brasil Redes.

A RUTE ${ }^{28}$, criada pelo Ministério da Ciência e Tecnologia, tem por objetivo conectar os hospitais universitários brasileiros entre si e com outros centros de excelência nacionais e internacionais. O Programa Nacional Teles- saúde Brasil Redes, criado pelo Ministério da Saúde, conecta alguns hospitais universitários à municípios remotos. Nesta rede brasileira dedicada à educação, pesquisa e serviço, prioritariamente, para os profissionais de saúde que atuam no Sistema Único de Saúde (SUS), estão inseridos diferentes grupos de interesse em radiologia e diagnóstico por imagem. Destacam-se os grupos de Residência em Radiologia30, Radiologia e Diagnóstico por Imagem do Tórax, Radiologia Diagnóstico por Imagem do Abdômen, Neurorradiologia e o de Radiologia e Diagnóstico em Pediatria (RADPED); este com maior abrangência e Residência em Radiologia ${ }^{30}$. Nesta rede globalizada em radiologia, diferentes metodologias são utilizadas: aulas por teleconferência com temas de atualização, seminários virtuais interativos, discussão de casos clínicos para treinamento profissional e discussão de casos de difícil diagnóstico para segunda-opinião entre grupos especializados. As reuniões são mensais, com acesso remoto pelo público-alvo composto por alunos de graduação e de pós-graduação em medicina, médicos generalistas e médicos radiologistas.

Alguns grupos de trabalho utilizam, também, a associação de duas tecnologias para teleconferência: a videoconferência e a webconferência, como o grupo RADPED, ampliando a abrangência das atividades ${ }^{9,10,12}$. Todos os eventos são gravados para serem reutilizados pelos grupos e há participantes em todos os estados brasileiros, incluindo hospitais universitários e municípios geograficamente afastados. Estes programas têm a certificação de qualidade acreditada pelas Universidades.

\section{Conclusão}

A telerradiologia é um recurso que contribui significativamente para a melhoria da qualidade da assistência médica, para a redução do tempo gasto entre o diagnóstico e a terapia, bem como para a extensão dos serviços 
médicos especializados e de qualidade para áreas deficientes em assistência ${ }^{1-9}$.

O Brasil, país com dimensões continentais, tem muito a ganhar com a formação e a consolidação de redes colaborativas integradas de educação e assistência médica a distância. Benefícios como a redução de deslocamentos de profissionais e de pacientes e a possibilidade de levar a Medicina especializada a regiões remotas e carentes do país fazem enorme diferença ${ }^{1-9}$.

Tanto o setor público quanto o privado, tem a ganhar, e muito, com esta ferramenta. A capacidade de ver e rever imagens, relatórios anteriores e manipular imagens sem perda de qualidade possibilitam maior acuidade diagnóstica, relatórios de melhor qualidade para o médico solicitante, alem de evitar reexposição à radiação. Em paralelo, a radiologia digital (PACS e RIS) reduz (ou elimina) o uso de químicos, ecologicamente incorretos. Para os médicos em geral, promove a inclusão digital, contribui na formação e atualização, bem como promove a integração multiprofissional e interdisciplinar. No tocante a gestão, reduz custos, aumenta o número de exames e qualifica o atendimento, uma vez que o tempo de atendimento, realização e retirada dos exames é drasticamente reduzido ${ }^{3}$.

A cada duas teleconsultorias oferecidas aos médicos e profissionais de saúde que atuam no SUS, evita-se a remoção de um paciente para atendimento em outro serviço. A teleconsultoria custa para o SUS oito vezes menos do que a remoção para atendimento em outro serviço ${ }^{31}$.

No futuro, certamente, surgirão outras tecnologias nacionais de baixo custo, tais como ScanRX3, utilizada em unidades básicas de saúde e unidades de pronto atendimento na cidade do Rio de Janeiro, onde as teleconsultorias realizadas em um período de seis meses, resultaram na detecção e encaminhamento de $13 \%$ de pacientes com critérios diagnósticos para tuberculose para centros de referência específica ${ }^{3}$.
A ciência médica e a engenharia tecnológica estão cada vez mais integradas e caminhando juntas a fim de proporcionarem recursos para a melhoria do sistema de saúde no mundo. O futuro da telerradiologia parece ser brilhante, pois todos os fatores são favoráveis. O desenvolvimento de novas soluções tecnológicas avançadas, como o da realidade virtual permitirá ações médicas cada vez mais complexas à distância.

A telerradiologia do amanhã indubitavelmente será reforçada pela robótica, como já acontece com o projeto datagloves (luvas de posicionamento digital) ${ }^{32}$. A melhoria e expansão da internet, a evolução dos equipamentos diagnósticos e a nanotecnologia farão da telerradiologia a ferramenta diagnóstica mais rápida e eficiente do mundo.

\section{Referências}

1. Lee SK, Peng CH, Wen CH, Huang SK, Jiang WZ. Consulting with Radiologists outside the Hospital by Using Java. Radiographics 1999;19:1069-75.

2. Nobre LF, von Wangenheim A, Maia RS, Ferreira L, Marchiori E. Certificação digital de exames em Telerradiologia: um alerta necessário. Radiol Bras 2007;40(6):415-21.

3. Monteiro AMV, Lima CMAO, Santos AAS. Global Teleradiology services and education: Brazilian projects to improve service and education. In: Feijó RA, Ziviani, Blanco PJ. Scientific Computting Applied to Medicine and Healthcare. Current State and Future Trends at the INCT-MACC the Brazilian National Institute of Science and Technology in Medicine Assisted by Scientific Computing. Petropólis, RJ: LNCC/MCTl;2012. p. 373-90.

4. El Khoury SG. Telemedicina: Análise de sua evolução no Brasil [dissertação de mestrado]. São Paulo: Universidade de São Paulo (USP);2003.

5. Lima CMAO, Monteiro AMV, Ribeiro EB, Portugal SM, Silva LSX, Junior MJ. Videoconferências: Sistematização e Experiências em Telemedicina. Radiol Bras 2007;40(5):341-4

6. Monteiro AMV, Corrêa DG, Santos AASMD, Cavalcanti SA, Sakuno T, Filgueiras T. Telemedicine and Pediatric Radiology: A New Environment for Training, Learning, and Interactive Discussions. Telemedicine Journal and e-Health, 2011;17:753-6.

7. Santos AASMD, Monteiro AMV, Messina LA, Nobre LF, Dippolito G. The use of Teleradiology to promote education and training of physicians and residents in radiology in Brazil. In: European Congress of Radiology, 2010, Viena. ESR 2010, 2010.

8. Santos AASMD, Monteiro AMV, Ziviani A, Feijó RA. MACC-Rio project: A network program of research and technical scientific cooperation between institutions in Brazil. In: European Congress of Radiology, 2010, Viena. ESR 2010, 2010.

9. Monteiro AMV, Santos AASMD, Cavalcanti S. The Brazilian Telemedicine pediatric radiology network. In: European Congress of Radiology, 2010, Viena. ESR 2010, 2010. 2004;113:639-43.

10. Monteiro AMV, Corrêa DG, Santos AASMD. Programa de Educação a Distância em Radiologia Pediátrica. In: Congre`s France Amérique Latine de Radiologie. 39a Jornada Paulista de Radiologia, 2009, São Paulo. Painéis e Temas Livres, 2009. p. 36. 
11. Santos AASMD, Monteiro AMV, Ziviani A. Participação da Radiologia no projeto MACC-Rio. In: Congre`s France Amérique Latine de Radiologie. 39a Jornada Paulista de Radiologia, 2009, São Paulo. Painéis e Temas Livres, 2009. p. 74

12. Monteiro AMV, Taborda M, Dias C. Rede RUTE Núcleo UERJ: Utilização de ambientes virtuais de aprendizagem como apoio a reutilização das teleconferências e a pesquisa colaborativa. In: IV Congresso Brasileiro de Telemedicina e Telessaúde, 2009, Belo Horizonte. IV Congresso Brasileiro de Telemedicina e Telessaúde. Belo Horizonte: UFMG, 2009 .

13. Monteiro AMV, João Junior M, Santos AASMD. Prospective Evaluation of a Teleducation Program by Videoconferencing to Enhace Radiology Resident Education. A Medical School of State University of Rio de Janeiro, Brazil, Teleteaching Initiative. In: 22nd ICDE World Conference on Distance Education, 2006, Rio de Janeiro. Promoting Quality in On-line Flexible and Distance Education. Rio de Janeiro: ABED, 2006.

14. Monteiro AMV. A Teleradiologia sem o PACS. O Projeto Inter-institucional entre Centro de Ensino Nacionais em Radiologia Pediátrica. In: $10^{\circ}$ Congress of the International Society for Telemedicine and e-health. 2nd Congress of Brazilian Council of Telemedicine and telehealth., 2005, São Paulo.SP. $10^{\circ}$ Congress of the International Society for Telemedicine and e-health. 2nd Congress of Brazilian Council of Telemedicine and telehealth., 2005. p. 15

15. Telerradiologia. Disponível em (http://pt.wikipedia.org/wiki/Telerradiologia). Acesso em 21 de novembro de 2012

16. Benefits of Teleradiology. Disponível em (http://www.corestudycast.com/news/ benefits-of-teleradiology). Acesso em 20 de fevereiro de 2013.

17. Hayward T, Mitchell J. The cost-effectiveness of teleradiology at the Women's and Children's Hospital in Adelaide. J Telemed Telecare 2000;6:23-5.

18. Bosmans JM, Peremans L, Menni M, De Schepper AM, Duyck PO, Parizel PM. Structured reporting: if, why, when, how-and at what expense? Results of a focus group meeting of radiology professionals from eight countries. Insights Imaging. 2012;3(3):295-302

19. McGill AF, North JB. Teleconference fracture clinics: a trial for rural hospitals. ANZ J Surg. 201;82(2):2-3

20. Colégio Brasileiro de Radiologia. Disponível em (http://www.cbr.org.br/). Acesso em 25 de novembro de 2012.
21. Cadastro Nacional de Estabelecimentos de Saúde. Disponível em (http://cnes.datasus. gov.br/Mod_Ind_Equipamento.asp?VEstado=00). Acesso em 29 de outubro de 2012.

22. Conselho Federal de Medicina. Resolução de Telerradiologia. Disponível em (http://www.portalmedico.org.br/resolucoes/CFM/2009/1890_2009.htm). Acesso em 5 de novembro de 2012

23. Novo Código de Ética Médica. Conselho Federal de Medicina. Disponível em (http:// www.portalmedico.org.br/novocodigo/index.asp). Acesso em 10 de outubro de 2012.

24. Conselho Federal de Medicina. Resolução de Telerradiologia. Disponível em (http://www.portalmedico.org.br/resolucoes/cfm/1992/1643_1992.htm). Acesso em 5 de novembro de 2012

25. American College of Radiology. ACR standart for teleradiology. Reston, Va: American College of Radiology, 1994

26. James H. Thrall. Teleradiology Part I. History and Clinical Applications. Radiology 2007:243:613-7.

27. Colégio Interamericano de Radiologia. Disponível em (http://www.webcir.org/ etica.php\#). Acesso em 9 de novembro de 2012.

28. Rede Universitária de Telemedicina (RUTE). Disponível em (http://rute.rnp.br/). Acesso em 19 de outubro de 2012.

29. Telessaúde Brasil. Disponível em (http://www.telessaudebrasil.org.br/). Acesso em 20 de novembro de 2012.

30. Rede Universitária de Telemedicina (RUTE) - Grupos de Interesse Especial. Disponível em (http://rute.rnp.br/sigs?p_p_id=buscasig\&p_p_lifecycle=0\&p_p

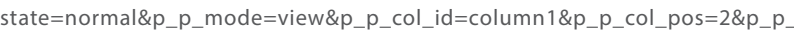
col_count $=3 \&$ buscasig_text $=\&$ buscasig_instituicaold $=1$ \&_buscasig_especialidadeld=\&_buscasig_struts_action=/rnp/sig/busca/view\&page=3). Acesso em 16 de outubro de 2012.

31. Telessaúde brasileiro é referência em atendimento. Disponivel em (http://portalsaude.saude.gov.br/portalsaude/noticia/3211/162/telessaude-brasileiro-e-referencia-em-atendimento.html). Acesso em 21 de fevereiro de 2013.

32. Cheng Y, Huang R. Using virtual reality environment to improve joint attention associated with pervasive developmental disorder. Res Dev Disabil. 2012;33(6):2141-52. 